\title{
ENUMERATING NEIGHBORS IN DIAMOND-LIKE LATTICES
}

by

\author{
A. V. M. Ferris-Prabhu \\ NASA Goddard Space Flight Center \\ Greenbelt, Maryland
}

In a previous paper ${ }^{1}$ a method was presented for determining the number and locations of neighbors of various order in non-Bravais lattices. In this note, a considerably simpler method which exploits the symmetry of the laticice is shown for diamond-like lattices.

The diamond lattice has a basis, and may be resolved into two face-centered sublattices, the edge of each being taken, for convenience, to be $4 \mathrm{a}$, where a is the lattice constant. Sublattice I is centered at $(0,0,0)$ and the origin of sublattice $I$, at $(1,1,1)$, is reached by translating through the basis vector

$$
\mathbf{b}=a_{1}+a_{2}+a_{3}
$$

where $a_{1}, a_{2}, a_{3}$ are three mutually orthogonal vectors, each of length a. (See Figure 1).

Sites on sublattices I and II, respectively, are located by the translation vectors

$$
\begin{aligned}
& r_{s}^{I}=2 l_{1} a_{1}+2 l_{2} a_{2}+2 l_{3} a_{3} \\
& r s=r \underset{s}{I I}+b
\end{aligned}
$$

\section{GPO PRICE}

\section{CFSTI PRICE(S)}

Hard copy (HC) $3 \cdot 00$

Microfiche (MF) 65

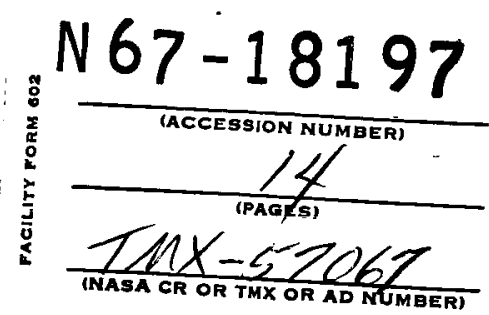




$$
2 \ell_{1}+2 l_{2}+2 \ell_{3}=4 \mathrm{~s}
$$

and

$$
\ell_{1}, \ell_{2}, \ell_{3}, s \text { are integers. }
$$

Equations (2) enable the following simple rules to be enunciated. The coordinates $(x, y, z)$ of sites on sublattice I are all even integers whose sum is exactly divisible by four.

$$
(x+y+z) / 4=\text { integer }
$$

The nearest neighbors of this site lie on sublattice II and their coordinates are $(x+1, y+1, z+1),(x+1, y-1, z-1),(x-1, y+1, z-1),(x-1, y-1$, $z+1)$

The coordinates $(x, y, z)$ of sites on sublattice II are all odd integers whose sum plus one, is exactly divisible by four.

$$
(x+y+z+1) / 4=\text { integer }
$$

The nearest neighbours of this site lie on sublattice I and their coordinates are $(x-1, y-1, z-1),(x-1, y+1, z+1),(x+1, y-1, z+1),(x+1, y+1, z-1)$

To enumerate and locate neighbours, one constructs shells of increasing radius and counts the number of lattice points lying on them. In the diamond structure, for a shell to have lattice points on it, it is seen from equations (2) that its radius must satisfy the relation 


$$
r_{n}^{2}= \begin{cases}4 n-1, & n 口 1,3,5, \cdots \\ 4 n & n=2,4,6, \cdots\end{cases}
$$

One then seeks integers $x, y, z$ such that

$$
x^{2}+y^{2}+z^{2}=r_{n}^{2}
$$

For $\mathrm{n}$ even, it is sufficient to find positive integers satisfying equation (5). Permuting the coordinates will give those sites in the first octant which lie on this shell. The total number of sites on the shell is obtained by multiplying each site in the first octant by the symmetry factor 2,4 , or 8 according to whether two, one or no coordinates arezero. (See Figures 2 and 3) e.g. $r_{14}^{2}=56=2^{2}+4^{2}$ $+6^{2}$. There are 6 sites in the first octant and 48 altogether.

For $\mathrm{n}$ odd, it is necessary to find odd integers which satisfy both equation (5) and equation (3b). By permuting the coordinates one obtains all sites on this shell. e.g. $r_{13}^{2}=51=1^{2}+1^{2}+7^{2}=1^{2}+5^{2}+5^{2}$. Triplets satisfying equations (5) and (3b) are $(1,1,-7)(1,-1,7),(-1,-1,-7),(1,5,5),(1,-5,-5),(-1,5,-5)$, from which it is clear that there are 24 such sites on this shell.

An electronic computer is necessary for very large $n$ and has been used to locate neighbours up to $\mathrm{n}=100$, but the advantage of this method is that for small $\mathrm{n}$, it provides a very quick and simple method of locating sites on either sublattice, and the number and distance of their neighbours. In Table I are listed values up to $\mathrm{n}=25$.

\section{ACKNOWLEDGMENTS}

I want to thank Mr. R. Hultberg for the excellent drawings, and Mr. E. Monasterski for writing a program to compute neighbours up to $n=100$. 
Table I. Neighbors of an atom in a diamond lattice

\begin{tabular}{|c|c|c|}
\hline Order $n$ & $(\text { Distance })^{2} \quad r_{n}^{2}$ & Number \\
\hline 1 & 3 & 4 \\
\hline 2 & 8 & 12 \\
\hline 3 & 11 & 12 \\
\hline 4 & 16 & 6 \\
\hline 5 & 19 & 12 \\
\hline 6 & 24 & 24 \\
\hline 7 & 27 & 16 \\
\hline 8 & 32 & 12 \\
\hline 9 & 35 & 24 \\
\hline 10 & 40 & 24 \\
\hline 11 & 43 & 12 \\
\hline 12 & 48 & 8 \\
\hline 13 & 51 & 24 \\
\hline 14 & 56 & 48 \\
\hline 15 & 59 & 36 \\
\hline 16 & 64 & 6 \\
\hline 17 & 67 & 12 \\
\hline 18 & 72 & 36 \\
\hline 19 & 75 & 28 \\
\hline 20 & 80 & 24 \\
\hline 21 & 83 & 36 \\
\hline 22 & 88 & 24 \\
\hline 23 & 91 & 24 \\
\hline 24 & 96 & 24 \\
\hline 25 & 99 & 36 \\
\hline
\end{tabular}

NOTE. $r_{n}^{2}$ may be likened to a one dimensional lattice with a basis. The periodicity is 8 and the basis is 3 . This is to be expected, as $\left|r_{\mathrm{s}}^{\mathrm{I}}\right|^{2}$ has periodicity 8 and $\mid r_{s}$ II $-\left.r_{s}^{I}\right|^{2}=|b|^{2}=3$. 


\section{LIST OF ILLUSTRATIONS}

Figure 1. The diamond lattice showing the two face-centered cubic sublattices related by the basis vector $b=a_{1}+a_{2}+a_{3}$.

Figure 2. Three sites in the first octant which have one coordinate equal to zero. Each site has a multiplicity factor of 4 .

Figure 3. Three sites in the first octant which have no coordinates equal to zero. Each site has a multiplicity factor of 8 .

\section{REFERENCE}

1. Van E. Wood, Am. J. Phys. $\underline{33}, 632$ (1965) . 


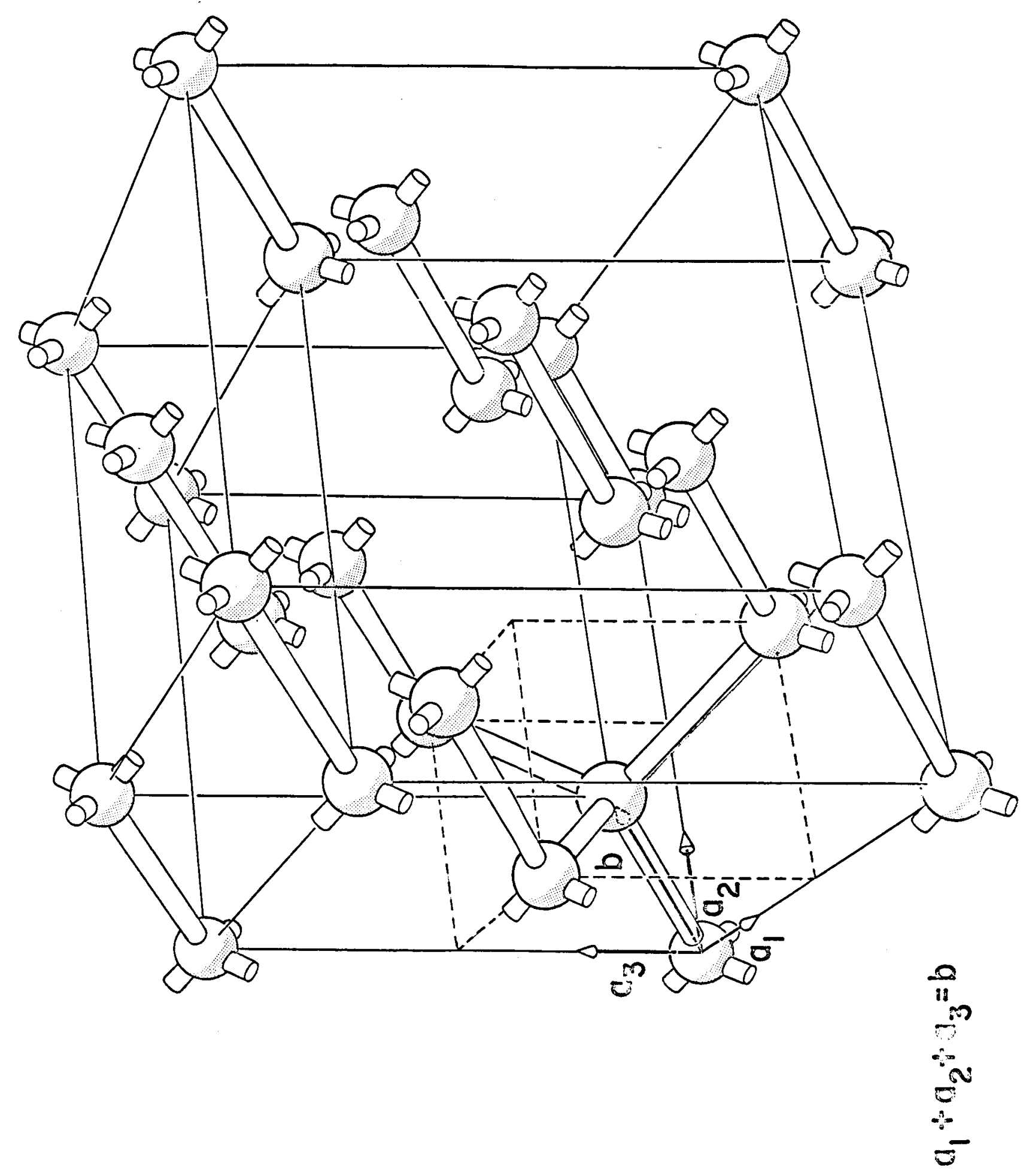




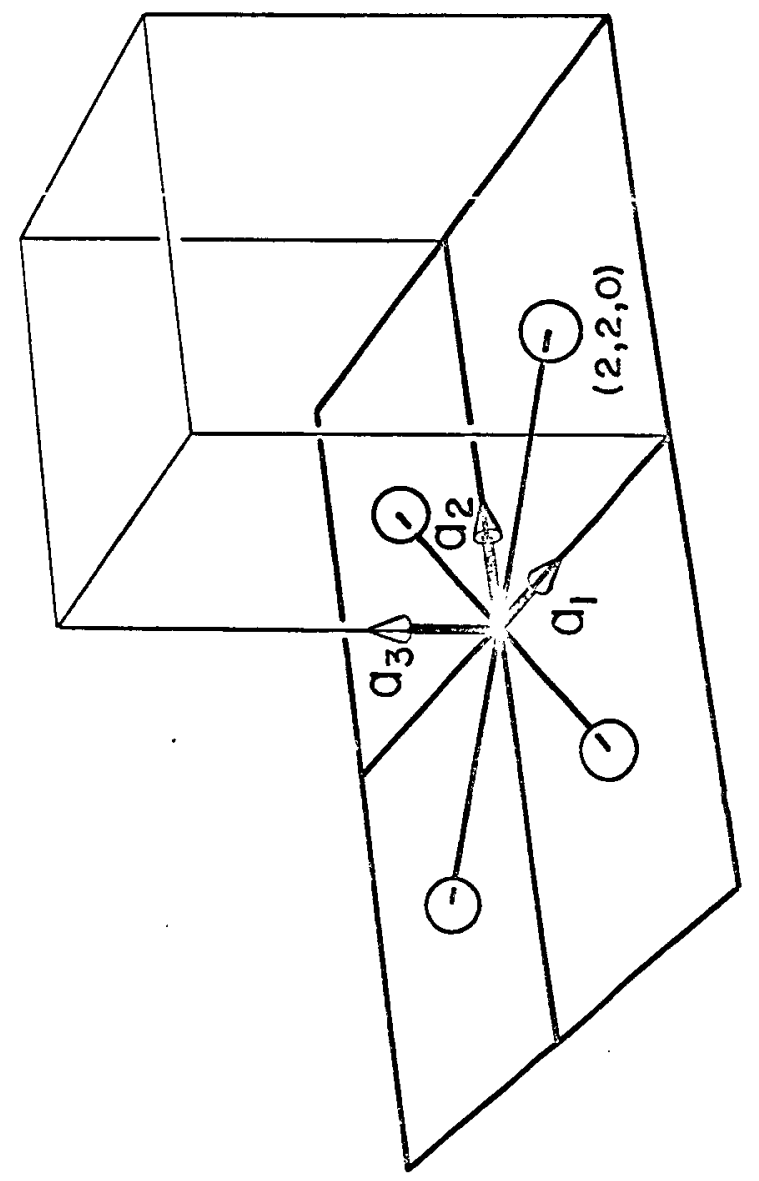




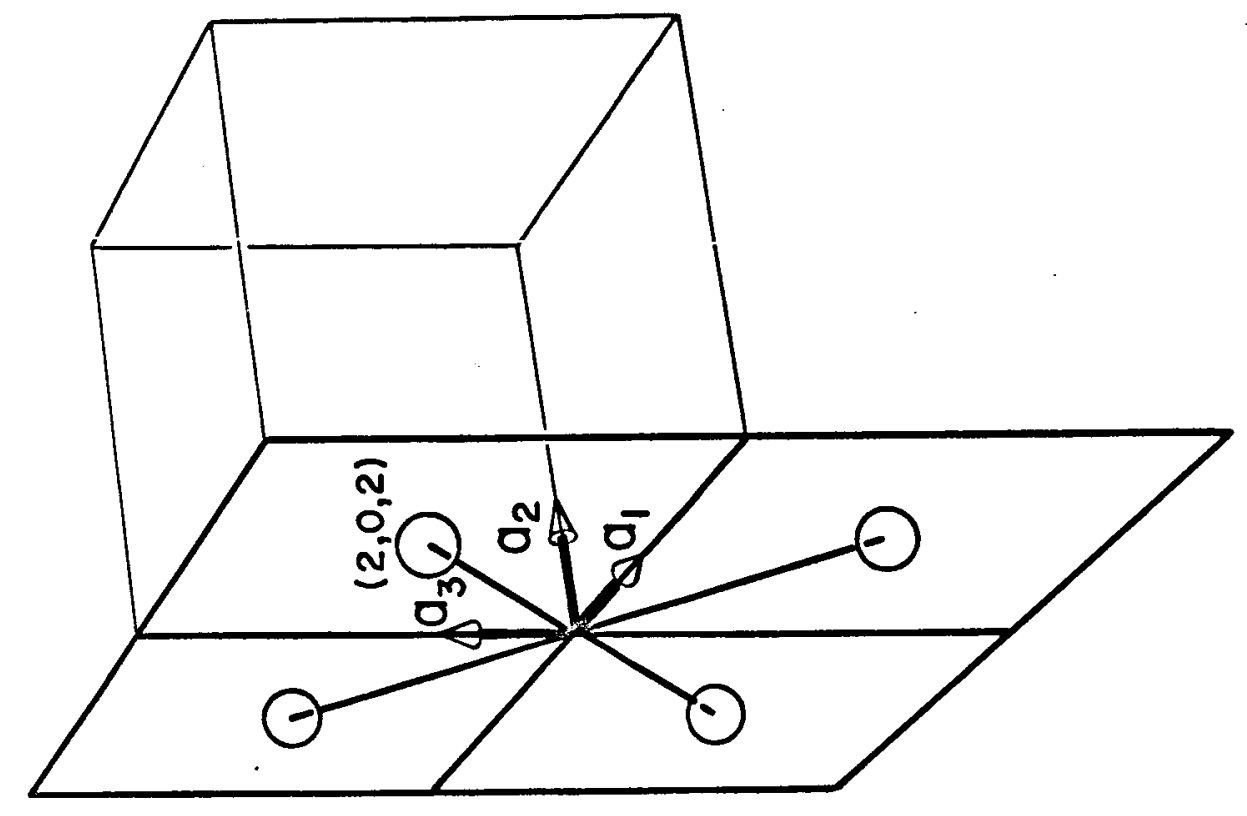




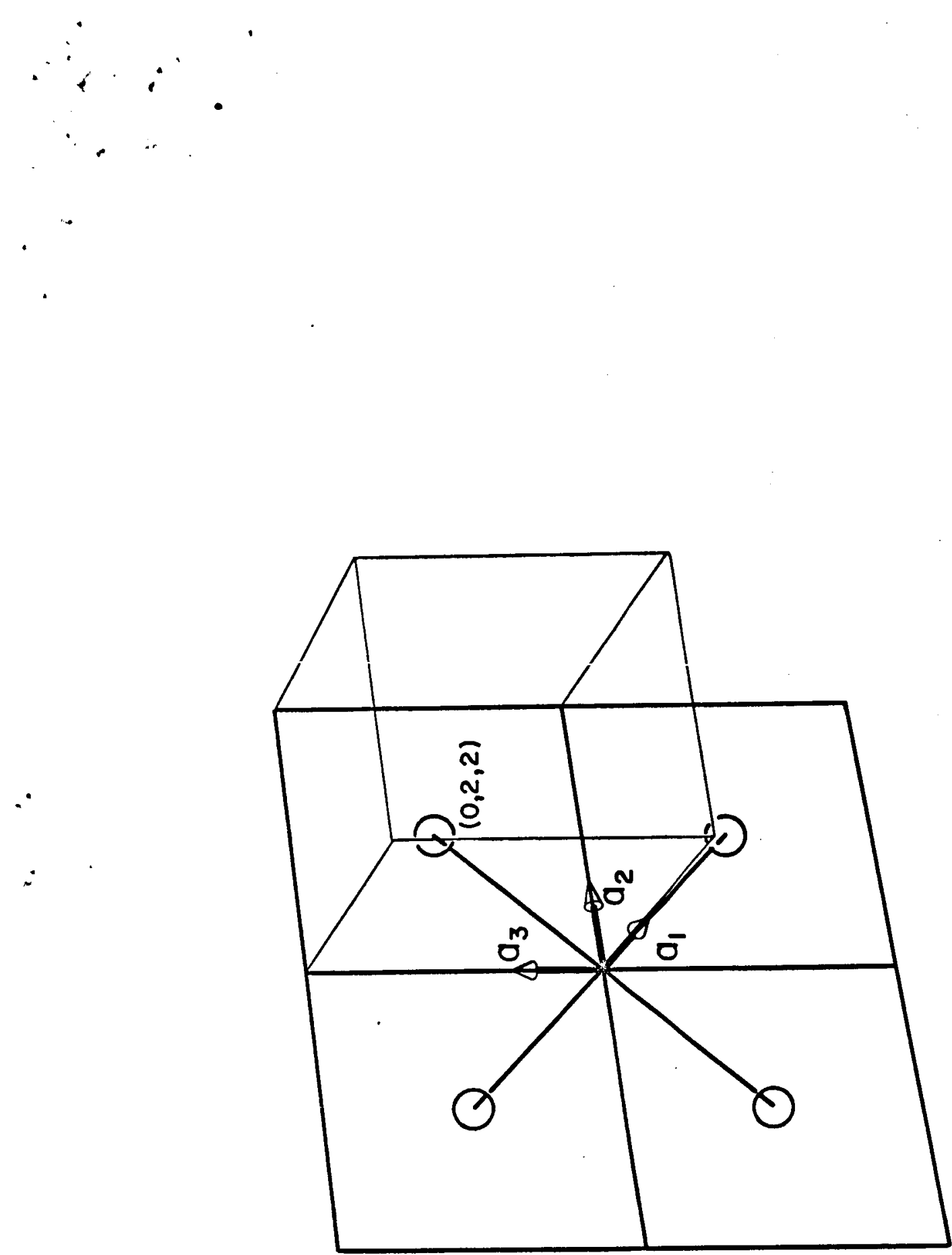


$\therefore \quad i$

$\therefore$

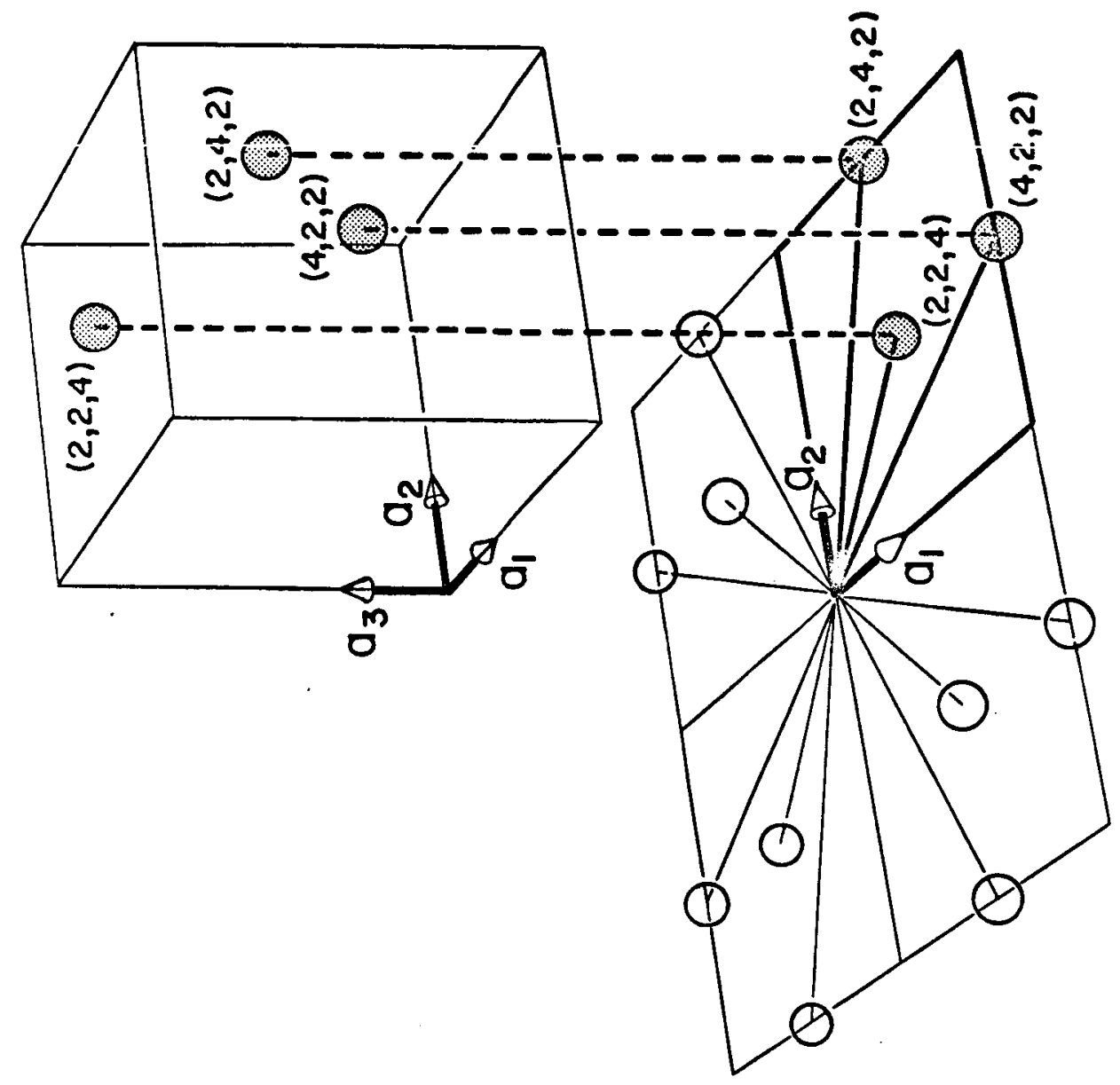

